Autonome Neuropathie des Gastrointestinaltrakts

\title{
Diabetes kann schwer im Magen liegen
}

\section{Typ-2-Diabetiker klagen häufig über unspezifische gastrointestinale Beschwerden. Bevor Sie eine Dyspepsie oder einen Reizdarm diagnosti- zieren, sollten Sie in diesen Fällen auch an eine autonome diabetische Neuropathie denken. Wie lässt sich die Diagnose sichern und welche Behandlungsmöglichkeiten gibt es?}

_ Über die Hälfte der Typ-2-Diabetiker klagt über gastrointestinale Symptome. Die Beschwerden reichen von Übelkeit, Erbrechen und Völlegefühl über Bauchschmerzen und Blähungen bis hin zu Obstipation, Diarrhoen und Stuhlinkontinenz. „Solche Symptome sind auch bei Nicht-Diabetikern weit verbreitet, was die Abgrenzung zur diabetischen Neuropathie schwer macht", sagte Dr. Hans Törnblom, Göteborg. Indizien sind gleichzeitig vorliegende Symptome einer kardialen oder urogenitalen autonomen Neuropathie. „Auch unzureichendes Ansprechen auf die oralen Antidiabetika, eine gestörte Hypoglykämiewahrnehmung und eine sonst nicht erklärbare Stoffwechsellabilität können auf eine Neuropathie hinweisen", so Törnblom.

\section{Schwierige Diagnostik}

Zum objektiven Nachweis einer Motilitätsstörung im Ösophagus dient die Manometrie. Vorher sollte zum Ausschluss einer anderen Erkrankung eine Endoskopie durchgeführt werden.

Für eine Gastropathie, die mit Völlegefühl, Erbrechen und vorzeitigem Sätti-

\section{Komplexe Pathogenese}

Pathogenetisch besteht eine Dysfunktion der neuralen Kontrolle von Motilität, Sekretion, Resorption und Perzeption, wobei eine funktionelle Schädigung afferenter und efferenter Nervenfasern des sympathischen und parasympathischen Nervensystems in jedem Organ des Gastrointestinaltrakts vorliegen kann. Häufig, aber nicht immer finden sich morphologische Schädigungen an den Nervenfasern. Diese sind durch toxisch-metabolische Einflüsse und/oder durch eine Mikroangiopathie verursacht. gungsgefühl einhergeht, spricht der Befund, dass 8-12 Stunden nach der letzten Nahrungsaufnahme endoskopisch noch Speisereste im Magen nachweisbar sind. Diagnostischer Goldstandard ist der szintigrafische Nachweis der gestörten Magenentleerung. Auch sonografisch, anhand der Veränderung der postprandialen Antrumfläche nach Aufnahme von $300 \mathrm{ml}$ Wasser, kann die verzögerte Magenentleerung objektiviert werden. „Ein einheitliches, allgemein anerkanntes diagnostisches Verfahren gibt es aber nicht", so Prof. Fernando Azpiroz, Barcelona.

Schwierig ist die Abklärung von Diarrhoen, da hier auch andere, bei Diabetikern häufig vorkommende Faktoren beteiligt sein können, z. B. Medikamente (Metformin), eine bakterielle Fehlbesiedlung oder eine exkretorische Pankreasinsuffizienz. Am aussagekräftigsten ist der $\mathrm{H}_{2}$-Atemtest nach Gabe von Glukose. Auch eine endoluminale Manometrie und eine Dünndarmszintigrafie sind möglich, aber sehr aufwendig.

Obstipation und Stuhlinkontinenz können beim Diabetiker vielerlei Ursachen haben. Deshalb sollte zunächst eine totale Koloskopie durchgeführt werden. Weitergehende Untersuchungen sind die Bestimmung der Kolontransitzeit mit röntgendichten Markern, die anorektale Manometrie, die Defäkografie und evtl. die Endosonografie.

\section{Therapeutische Möglichkeiten}

Die Symptome bei der diabetischen Gastropathie lassen sich oft schon mit diätetischen Maßnahmen günstig beeinflussen. Empfehlenswert sind mehrere kleinere Mahlzeiten über den Tag verteilt mit wenig Ballaststoffen.

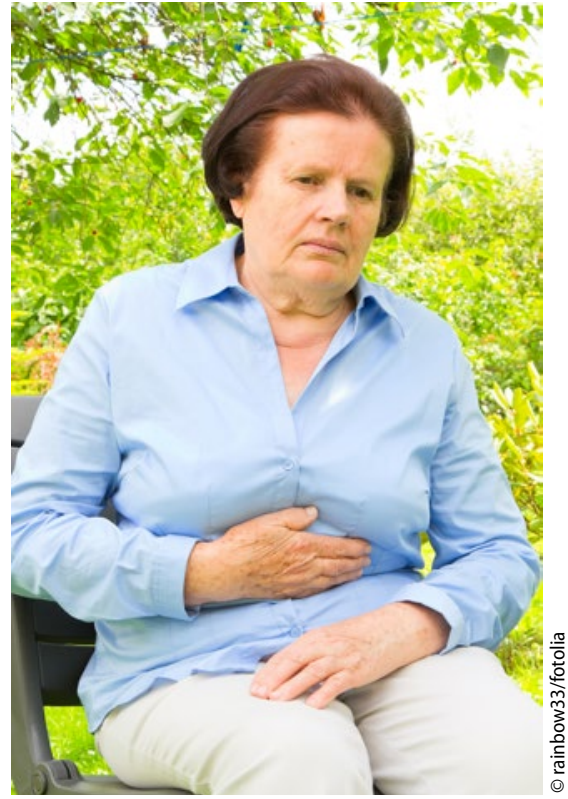

Kommt ihr Unwohlsein vom Diabetes?

Als gastroprokinetisch wirksame Pharmaka stehen Metoclopramid, Domperidon und Erythromycin zur Verfügung. Domperidon ist gleich effektiv wie Metoclopramid, zeigt jedoch keine zentralnervösen Nebenwirkungen. Erythromycin eignet sich nicht für eine Langzeittherapie, da sich rasch eine Toleranz entwickelt. Erythromycinanaloga, die nur die Motilinrezeptoren stimulieren, sind in der Entwicklung.

Bei Versagen der medikamentösen Therapie sollte die Implantation eines gastralen Schrittmachers diskutiert werden. In sehr schweren Fällen kann auch die Anlage einer Magensonde oder eine Gastrojejunostomie nötig werden.

Bei Diarrhö empfehlen sich die Gabe von Loperamid und bei Nachweis einer bakteriellen Fehlbesiedlung Antibiotika. Nach Absetzen der antibiotischen Therapie rezidivieren die Symptome allerdings rasch. Bei hartnäckiger Obstipation sind Laxanzien unverzichtbar. Hier sollte volumenfördernden Substanzen der Vorzug gegeben werden.

- Dr. med. Peter Stiefelhagen

- Quelle: 51. Jahrestagung der European Association for the Study in Diabetes, Stockholm, 14.-18.9.2015 